\title{
Safety Huddle Intervention for Reducing Physiologic Monitor Alarms: A Hybrid Effectiveness-Implementation Cluster Randomized Trial
}

\author{
Christopher P. Bonafide, MD, MSCE ${ }^{1,2,3,4 *}$, A. Russell Localio, PhD5 , Shannon Stemler, BA 1,3,6, Luis Ahumada, MSCS, PhD7, \\ Maya Dewan, MD, $\mathrm{MPH}^{8}$, Elizabeth Ely, RN, $\mathrm{PhD}^{9}$, Ron Keren, $\mathrm{MD}, \mathrm{MPH}^{1,2,3,4}$
}

\begin{abstract}
${ }^{1}$ Division of General Pediatrics, Children's Hospital of Philadelphia, Philadelphia, Pennsylvania; ${ }^{2}$ Department of Pediatrics, Perelman School of Medicine at the University of Pennsylvania, Philadelphia, Pennsylvania; ${ }^{3}$ Center for Pediatric Clinical Effectiveness, Children's Hospital of Philadelphia, Philadelphia, Pennsylvania; ${ }^{4}$ Leonard Davis Institute of Health Economics, University of Pennsylvania, Philadelphia, Pennsylvania; ${ }^{5}$ Department of Biostatistics and Epidemiology, Perelman School of Medicine at the University of Pennsylvania, Philadelphia, Pennsylvania; ${ }^{6}$ University of Pennsylvania School of Nursing, Philadelphia, Pennsylvania; ${ }^{7}$ Enterprise Analytics and Reporting, Children's Hospital of Philadelphia, Philadelphia, Pennsylvania; ${ }^{8}$ Division of Critical Care Medicine, Cincinnati Children's Hospital Medical Center, Cincinnati, OH; ${ }^{9}$ Department of Nursing, Children's Hospital of Philadelphia, Philadelphia, Pennsylvania.
\end{abstract}

BACKGROUND: Monitor alarms occur frequently but rarely warrant intervention.

OBJECTIVE: This study aimed to determine if a safety huddle-based intervention reduces unit-level alarm rates or alarm rates of individual patients whose alarms are discussed, as well as evaluate implementation outcomes.

DESIGN: Unit-level, cluster randomized, hybrid effectiveness-implementation trial with a secondary patient-level analysis.

SETTING: Children's hospital.

PATIENTS: Unit-level: all patients hospitalized on four control $(n=4177)$ and four intervention $(n=$ 7131) units between June 15, 2015 and May 8, 2016.

Patient-level: 425 patients on randomly selected dates postimplementation.

INTERVENTION: Structured safety huddle review of alarm data from the patients on each unit with the most alarms, with a discussion of ways to reduce alarms.

MEASUREMENTS: Unit-level: change in unit-level alarm rates between baseline and postimplementation periods in intervention versus control units. Patient-level: change in individual patients' alarm rates between the 24 hours leading up to huddles and the 24 hours after huddles in patients who were discussed versus not discussed in huddles.

RESULTS: Alarm data informed 580 huddle discussions. In unit-level analysis, intervention units had 2 fewer alarms/patient-day (95\% Cl: 7 fewer to 6 more, $P=.50$ ) compared with control units. In patient-level analysis, patients discussed in huddles had 97 fewer alarms/patientday $(95 \% \mathrm{Cl}: 52-138$ fewer, $P<.001)$ in the posthuddle period compared with patients not discussed in huddles. Implementation outcome analysis revealed a low intervention dose of 0.85 patients/unit/day.

CONCLUSIONS: Safety huddle-based alarm discussions did not influence unit-level alarm rates due to low intervention dose but were effective in reducing alarms for individual children. Journal of Hospital Medicine 2018:13:609-615. Published online first February 27, 2018. (C) 2018 Society of Hospital Medicine hysiologic monitor alarms occur frequently in the hospital environment, with average rates on pediatric wards between 42 and 155 alarms per monitored patient-day. ${ }^{1}$ However, average rates do not depict the full story, because only $9 \%-25 \%$ of patients are responsible for most alarms on inpatient wards. ${ }^{1,2}$ In addition, only $0.5 \%-1 \%$

\footnotetext{
*Address for correspondence: Christopher P. Bonafide, MD, MSCE, Children's Hospital of Philadelphia, 34th St and Civic Center Blvd, Suite 12NW80, Philadelphia, PA 19104; Telephone: 267-426-2901; Email: bonafide@email.chop.edu

Additional Supporting Information may be found in the online version of this article.
}

Received: November 2, 2017; Revised: December 27, 2017;

Accepted: January 6, 2018

๑) 2018 Society of Hospital Medicine DOI 10.12788/jhm.2956 of alarms on pediatric wards warrant action., ${ }^{3,4}$ Downstream consequences of high alarm rates include interruptions ${ }^{5,6}$ and alarm fatigue. ${ }^{3,4,7}$

Alarm customization, the process of reviewing individual patients' alarm data and using that data to implement patient-specific alarm reduction interventions, has emerged as a potential approach to unit-wide alarm management. ${ }^{8-11}$ Potential customizations include broadening alarm thresholds, instituting delays between the time the alarm condition is met and the time the alarm sounds, and changing electrodes. ${ }^{8-11} \mathrm{How}-$ ever, the workflows within which to identify the patients who will benefit from customization, make decisions about how to customize, and implement customizations have not been delineated.

Safety huddles are brief structured discussions among physicians, nurses, and other staff aiming to identify and mitigate threats to patient safety. ${ }^{11-13}$ In this study, we aimed to evaluate 


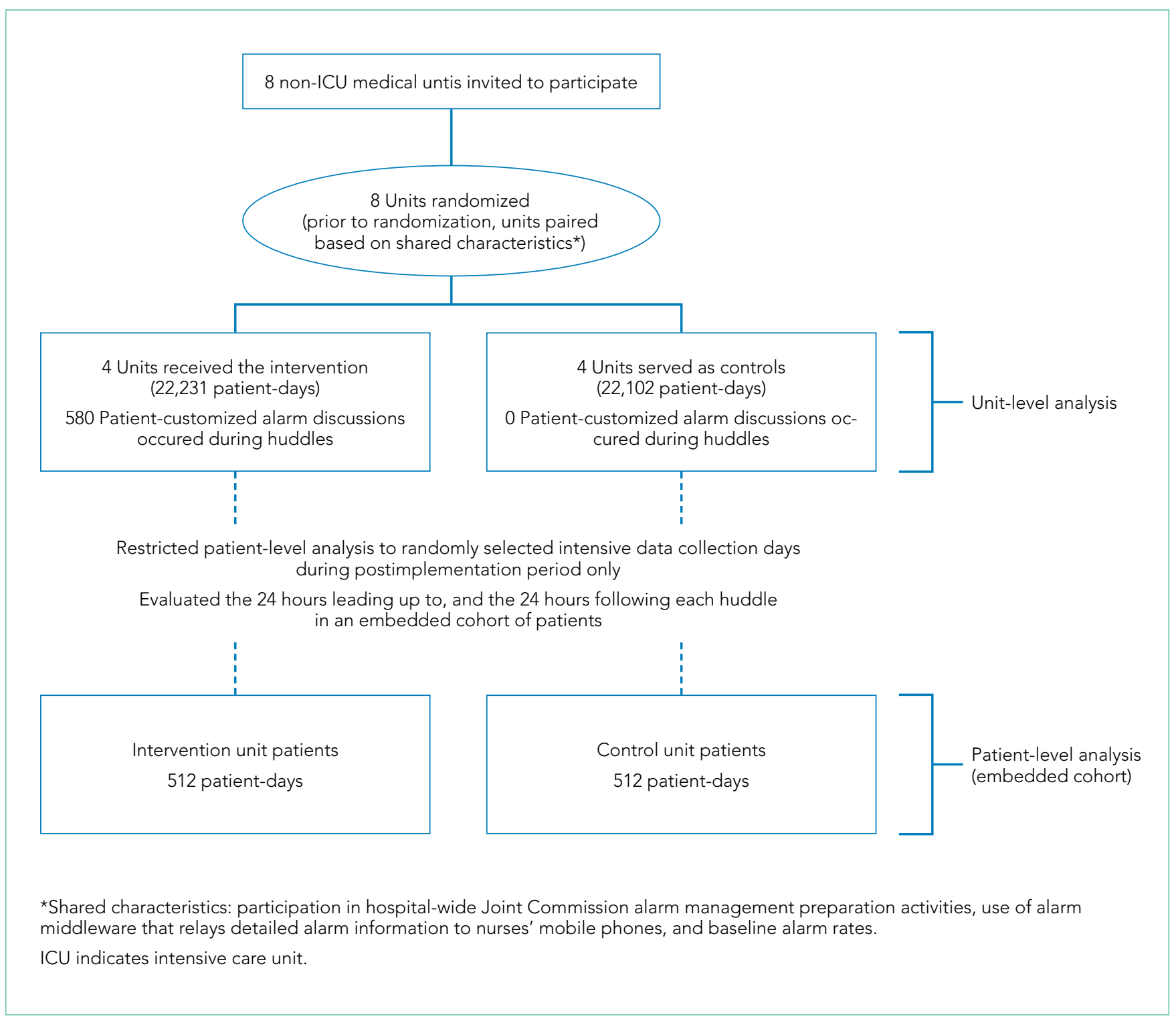

FIG 1. CONSORT flow diagram.

the influence of a safety huddle-based alarm intervention strategy targeting high alarm pediatric ward patients on (a) unit-level alarm rates and (b) patient-level alarm rates, as well as to (c) evaluate implementation outcomes. We hypothesized that patients discussed in huddles would have greater reductions in alarm rates in the 24 hours following their huddle than patients who were not discussed. Given that most alarms are generated by a small fraction of patients, ${ }^{1,2}$ we hypothesized that patient-level reductions would translate to unit-level reductions.

\section{METHODS}

\section{Human Subject Protection}

The Institutional Review Board of Children's Hospital of Philadelphia approved this study with a waiver of informed consent. We registered the study at ClinicalTrials.gov (identifier NCT02458872). The original protocol is available as an Online Supplement.

\section{Design and Framework}

We performed a hybrid effectiveness-implementation trial at a single hospital with cluster randomization at the unit level (CONSORT flow diagram in Figure 1). Hybrid trials aim to determine the effectiveness of a clinical intervention (alarm customization) and the feasibility and potential utility of an implementation strategy (safety huddles). ${ }^{14}$ We used the Consolidated Framework for Implementation Research ${ }^{15}$ to theoretically ground and frame our implementation and drew upon the work of Proctor and colleagues ${ }^{16}$ to guide implementation outcome selection.

For our secondary effectiveness outcome evaluating the effect of the intervention on the alarm rates of the individual patients discussed in huddles, we used a cohort design embedded within the trial to analyze patient-specific alarm data collected only on randomly selected "intensive data collection days," described below and in Figure 1. 


\section{Setting and Subjects}

All patients hospitalized on eight units that admit general pediatric and medical subspecialty patients at Children's Hospital of Philadelphia between June 15, 2015 and May 8, 2016 were included in the primary (unit-level) analysis. Every patient's bedside included a General Electric Dash 3000 physiologic monitor. Decisions to monitor patients were made by physicians and required orders. Default alarm settings are available in Supplementary Table 1; these settings required orders to change.

All eight units were already convening scheduled safety huddles led by the charge nurse each day. All nurses and at least one resident were expected to attend; attending physicians and fellows were welcome but not expected to attend. Huddles focused on discussing safety concerns and patient flow. None of the preexisting huddles included alarm discussion.

\section{Intervention}

For each nonholiday weekday, we generated customized paper-based alarm huddle data "dashboards" (Supplementary Figure 1) displaying data from the patients (up to a maximum of four) on each intervention unit with the highest numbers of high-acuity alarms ("crisis" and "warning" audible alarms, see Supplementary Table 2 for detailed listing of alarm types) in the preceding four hours by reviewing data from the monitor network using BedMasterEx v4.2 (Excel Medical Electronics, Jupiter, Florida). Dashboards listed the most frequent types of alarms, alarm settings, and included a script for discussing the alarms with checkboxes to indicate changes agreed upon by the team during the huddle. Patients with fewer than 20 alarms in the preceding four hours were not included; thus, sometimes fewer than four patients' data were available for discussion. We hand-delivered dashboards to the charge nurses leading huddles, and they facilitated the multidisciplinary alarm discussions focused on reviewing alarm data and customizing settings to reduce unnecessary alarms.

\section{Study Periods}

The study had 3 periods as shown in Supplementary Figure 2: (1) 16-week baseline data collection, (2) phased intervention implementation during which we serially spent 2-8 weeks on each of the four intervention units implementing the intervention, and (3) 16-week postimplementation data collection.

\section{Outcomes}

The primary effectiveness outcome was the change in unit-level alarms per patient day between the baseline and postimplementation periods in intervention versus control units, with all patients on the units included. The secondary effectiveness outcome (analyzed using the embedded cohort design) was the change in individual patient-level alarms between the 24 hours leading up to a huddle and the 24 hours following huddles in patients who were versus patients who were not discussed in huddles.

Implementation outcomes included adoption and fidelity measures. To measure adoption (defined as "intention to try" the intervention), ${ }^{16}$ we measured the frequency of discussions attended by patients' nurses and physicians. We evaluated three elements of fidelity: adherence, dose, and quality of delivery. ${ }^{17}$ We measured adherence as the incorporation of alarm discussion into huddles when there were eligible patients to discuss. We measured dose as the average number of patients discussed on each unit per calendar day during the postimplementation period. We measured quality of delivery as the extent to which changes to monitoring that were agreed upon in the huddles were made at the bedside.

\section{Safety Measures}

To surveil for unintended consequences of reduced monitoring, we screened the hospital's rapid response and code blue team database weekly for any events in patients previously discussed in huddles that occurred between huddle and hospital discharge. We reviewed charts to determine if the events were related to the intervention.

\section{Randomization}

Prior to randomization, the eight units were divided into pairs based on participation in hospital-wide Joint Commission alarm management activities, use of alarm middleware that relayed detailed alarm information to nurses' mobile phones, and baseline alarm rates. One unit in each pair was randomized to intervention and the other to control by coin flip.

\section{Data Collection}

We used Research Electronic Data Capture (REDCap) ${ }^{18}$ database tools.

\section{Data for Unit-Level Analyses}

We captured all alarms occurring on the study units during the study period using data from BedMasterEx. We obtained census data accurate to the hour from the Clinical Data Warehouse.

\section{Data Captured in All Huddles}

During each huddle, we collected the number of patients whose alarms were discussed, patient characteristics, presence of nurses and physicians, and monitoring changes agreed upon. We then followed up four hours later to determine if changes were made at the bedside by examining monitor settings.

\section{Data Captured Only During Intensive Data Collection Days} We randomly selected one day during each of the 16 weeks of the postimplementation period to obtain additional patient-level data. On each intensive data collection day, the four monitored patients on each intervention and control unit with the most high-acuity alarms in the four hours prior to huddles occurring - regardless of whether or not these patients were later discussed in huddles - were identified for data collection. On these dates, a member of the research team reviewed each patient's alarm counts in four-hour blocks during the 24 hours before and after the huddle. Given that the huddles were not always at the same time every day (ranging between 10:00 AM and 1:00 PM), we operationally set the huddle time as 12:00 PM for all units. 
TABLE 1. Alarm Rates for Intervention and Control Units Across All Study Periods from Interrupted Time Series Piecewise Regression Analysis

\begin{tabular}{|c|c|c|c|c|}
\hline & Control Units & Intervention Units & Difference in Rate Differences & Difference in Differences $P$ Value \\
\hline Units (clusters) & 4 & 4 & & \\
\hline Patient-days & 22,102 & 22,231 & & \\
\hline Unique patients & 4177 & 7131 & & \\
\hline Baseline alarms/patient-day (95\% CI) & $77(49-115)$ & $46(32-59)$ & & \\
\hline $\begin{array}{l}\text { Phased implementation alarms/patient-day } \\
(95 \% \mathrm{Cl})\end{array}$ & $76(51-107)$ & $53(37-71)$ & & \\
\hline Postimplementation alarms/patient-day (95\% CI) & $85(57-117)$ & $52(40-65)$ & & \\
\hline $\begin{array}{l}\text { Rate difference in alarms/patient-day }(95 \% \mathrm{Cl}) \text { : } \\
\text { Baseline versus postimplementation }\end{array}$ & $+8(+2$ to +14$)$ & $+6(+5$ to +9$)$ & $-2(-7$ to +6$)$ & .50 \\
\hline
\end{tabular}

Abbreviation: $\mathrm{Cl}$, confidence interval.

Data Analysis

We used Stata/SE 14.2 for all analyses.

\section{Unit-Level Alarm Rates}

To compare unit-level rates, we performed an interrupted time series analysis using segmented (piecewise) regression to evaluate the impact of the intervention. ${ }^{19,20}$ We used a multivariable generalized estimating equation model with the negative binomial distribution ${ }^{21}$ and clustering by unit. We bootstrapped the model and generated percentile-based $95 \%$ confidence intervals. We then used the model to estimate the alarm rate difference in differences between the baseline data collection period and the postimplementation data collection period for intervention versus control units.

\section{Patient-Level Alarm Rates}

In contrast to unit-level analysis, we used an embedded cohort design to model the change in individual patients' alarms between the 24 hours leading up to huddles and the 24 hours following huddles in patients who were versus patients who were not discussed in huddles. The analysis was restricted to the patients included in intensive data collection days. We performed bootstrapped linear regression and generated percentile-based 95\% confidence intervals using the difference in four-hour block alarm rates between pre- and posthuddle as the outcome. We clustered within patients. We stratified by unit and preceding alarm rate. We modeled the alarm rate difference between the 24-hour prehuddle and the 24-hour posthuddle for huddled and nonhuddled patients and the difference in differences between exposure groups.

\section{Implementation Outcomes}

We summarized adoption and fidelity using proportions.

\section{RESULTS}

Alarm dashboards informed 580 structured alarm discussions during 353 safety huddles (huddles often included discussion of more than one patient).

\section{Unit-Level Alarm Rates}

A total of 2,874,972 alarms occurred on the eight units during the study period. We excluded 15,548 alarms that occurred during the same second as another alarm for the same patient because they generated a single alarm. We excluded 24,700 alarms that occurred during 4 days with alarm database downtimes that affected data integrity. Supplementary Table 2 summarizes the characteristics of the remaining 2,834,724 alarms used in the analysis.

Visually, alarm rates over time on each individual unit appeared flat despite the intervention (Supplementary Figure 3). Using piecewise regression, we found that intervention and control units had small increases in alarm rates between the baseline and postimplementation periods with a nonsignificant difference in these differences between the control and intervention groups (Table 1).

\section{Patient-Level Alarm Rates}

We then restricted the analysis to the patients whose data were collected during intensive data collection days. We obtained data from 1974 pre-post pairs of four-hour time periods.

Patients on intervention and control units who were not discussed in huddles had 38 fewer alarms/patient-day $(95 \% \mathrm{Cl}$ : 23-54 fewer, $P<.001)$ in the posthuddle period than in the prehuddle period. Patients discussed in huddles had 135 fewer alarms/patient-day (95\% Cl: 93-178 fewer, $P<.001)$ in the posthuddle 24-hour period than in the prehuddle period. The pairwise comparison reflecting the difference in differences showed that huddled patients had a rate of 97 fewer alarms/ patient-day (95\% Cl: 52-138 fewer, $P<.001)$ in the posthuddle period compared with patients not discussed in huddles.

To better understand the mechanism of reduction, we analyzed alarm rates for the patient categories shown in Table 2 and 


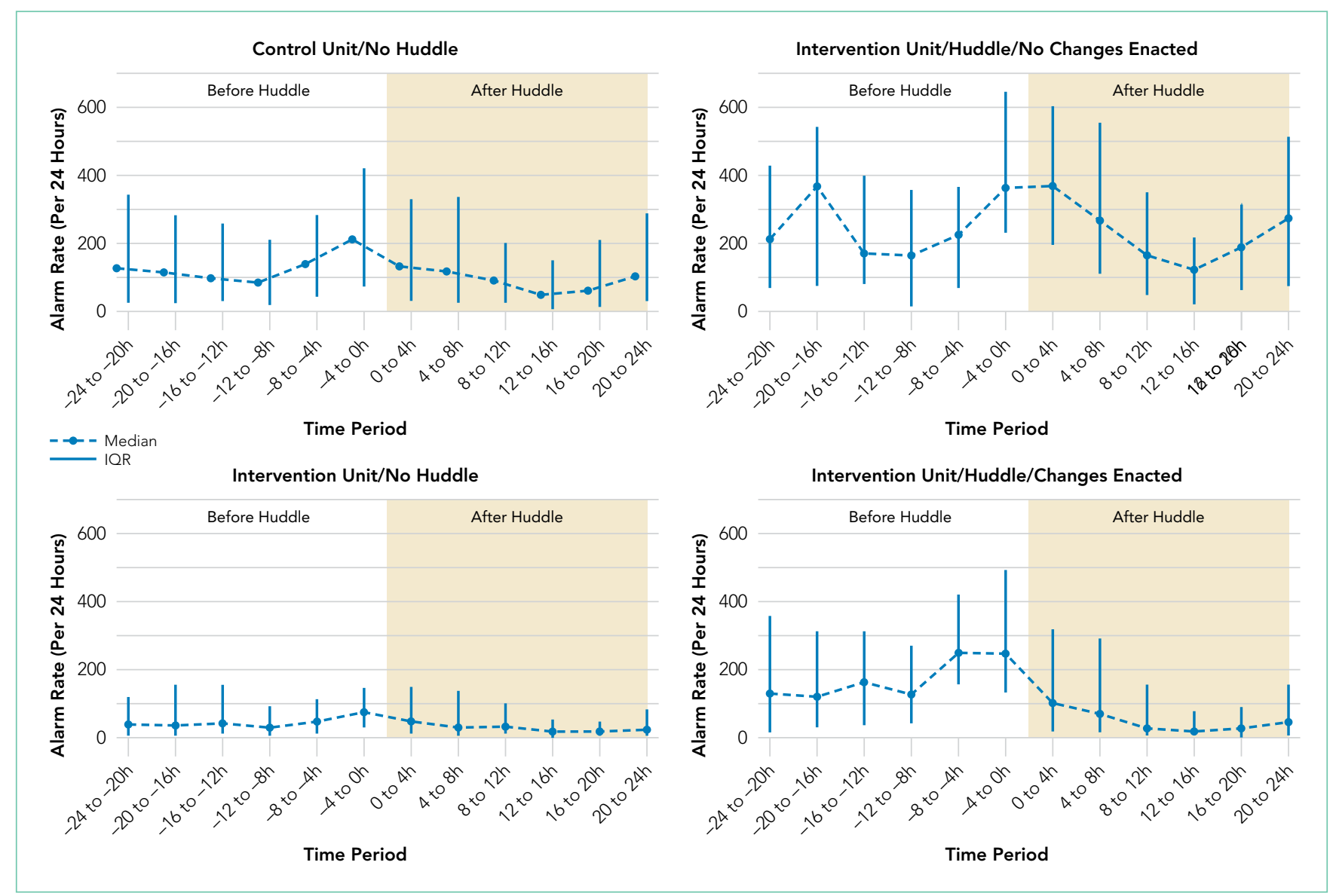

FIG 2. Patient-level alarm rates in the 24 hours leading up to safety huddles and the 24 hours after huddles. Abbreviation: IQR, interquartile range.

visually evaluated how average alarm rates changed over time (Figure 2). When analyzing the six potential pairwise comparisons between each of the four categories separately, we found that the following two comparisons were statistically significant: (1) patients whose alarms were discussed in huddles and had changes made to monitoring had greater alarm reductions than patients on control units, and (2) patients whose alarms were discussed in huddles and had changes made to monitoring had greater alarm reductions than patients who were also on intervention units but whose alarms were not discussed (Table 2).

\section{Implementation Outcomes}

\section{Adoption}

The patient's nurse attended 482 of the 580 huddle discussions $(83.1 \%)$, and at least one of the patient's physicians (resident, fellow, or attending) attended 394 (67.9\%).

\section{Fidelity: Adherence}

In addition to the 353 huddles that included alarm discussion, 123 instances had no patients with $\geq 20$ high acuity alarms in the preceding 4 hours therefore, no data were brought to the huddle. There were an additional 30 instances when a huddle did not occur or there was no alarm discussion in the huddle despite data being available. Thus, adherence occurred in 353 of 383 huddles (92.2\%).

\section{Fidelity: Dose}

During the 112 calendar day postimplementation period, 379 patients' alarms were discussed in huddles for an average intervention dose of 0.85 discussions per unit per calendar day.

\section{Fidelity: Quality of Delivery}

In 362 of the 580 huddle discussions (62.4\%), changes were agreed upon. The most frequently agreed upon changes were discontinuing monitoring (32.0\%), monitoring only when asleep or unsupervised $(23.8 \%)$, widening heart rate parameters (12.7\%), changing electrocardiographic leads/wires (8.6\%), changing the pulse oximetry probe (8.0\%), and increasing the delay time between when oxygen desaturation was detected and when the alarm was generated (4.7\%). Of the huddle discussions with changes agreed upon, 346 (95.6\%) changes were enacted at the bedside.

\section{Safety Measures}

There were zero code blue events and 26 rapid response team activations for patients discussed in huddles. None were related to the intervention.

\section{DISCUSSION}

Our main finding was that the huddle strategy was effective in safely reducing the burden of alarms for the high alarm pedi- 
TABLE 2. Alarm Rate Differences Based on Patient Category

\begin{tabular}{|c|c|c|c|c|}
\hline & $\begin{array}{c}\text { Category } 1 . \\
\text { Control Unit, } \\
\text { Not Discussed in Huddle }\end{array}$ & $\begin{array}{c}\text { Category } 2 . \\
\text { Intervention Unit, } \\
\text { Not Discussed in Huddle }\end{array}$ & $\begin{array}{c}\text { Category } 3 . \\
\text { Intervention Unit, } \\
\text { Discussed in Huddle, } \\
\text { But No Changes Made in } 4 \mathrm{~h} \\
\text { After Huddle }\end{array}$ & $\begin{array}{c}\text { Category } 4 . \\
\text { Intervention Unit, } \\
\text { Discussed in Huddle, } \\
\text { Monitor Changes Made in } 4 \mathrm{~h} \\
\text { After Huddle }\end{array}$ \\
\hline Huddles or huddle opportunities, $n^{\text {a }}$ & 256 & 135 & 34 & 87 \\
\hline Unique patients, n & 201 & 126 & 27 & 71 \\
\hline $\begin{array}{l}\text { Pre/post-huddle difference in alarms/ } \\
\text { patient-day }(95 \% \mathrm{Cl})\end{array}$ & 49 fewer ( 29 to 70 fewer) & 14 fewer (35 fewer to 11 more) & 54 fewer (155 fewer to 31 more) & 168 fewer (125 to 217 fewer) \\
\hline \multicolumn{5}{|l|}{ Versus Category 1: } \\
\hline $\begin{array}{l}\text { Difference in differences contrast in } \\
\text { alarms/patient-day }(95 \% \mathrm{Cl}, \mathrm{P})^{\mathrm{b}}\end{array}$ & & $\begin{array}{l}35 \text { more (7 fewer to } 78 \text { more, } \\
\qquad P=.17)\end{array}$ & $\begin{array}{l}5 \text { fewer (130 fewer to } 121 \text { more, } \\
\qquad P=.99)\end{array}$ & $\begin{array}{l}119 \text { fewer (186 fewer to } 52 \text { fewer, } \\
\qquad P<.001)\end{array}$ \\
\hline \multicolumn{5}{|l|}{ Versus Category 3: } \\
\hline $\begin{array}{l}\text { Difference in differences contrast in } \\
\text { alarms/patient-day }(95 \% \mathrm{Cl}, \mathrm{P})^{\mathrm{b}}\end{array}$ & & & & $\begin{array}{l}114 \text { fewer (253 fewer to } 24 \text { more, } \\
\qquad P=.17 \text { ) }\end{array}$ \\
\hline \multicolumn{5}{|c|}{$\begin{array}{l}\text { aPatients who were not discussed in huddles (Groups } 1 \text { and 2) but whose data we obtained for comparison on intensive data collection days are enumerated here as "huddle opportunities." } \\
\text { 'Cl and P value adjusted for } 6 \text { pairwise comparisons using Bonferroni method. }\end{array}$} \\
\hline
\end{tabular}

atric ward patients whose alarms were discussed, but it did not reduce unit-level alarm rates. Implementation outcomes explained this finding. Although adoption and adherence were high, the overall dose of the intervention was low.

We also found that $36 \%$ of alarms had technical causes, the majority of which were related to the pulse oximetry probe detecting that it was off the patient or searching for a pulse. Although these alarms are likely perceived differently by clinical staff (most monitors generate different sounds for technical alarms), they still represent a substantial contribution to the alarm environment. Minimizing them in patients who must remain continuously monitored requires more intensive effort to implement other types of interventions than the main focus of this study, such as changing pulse oximetry probes and electrocardiographic leads/wires.

In one-third of huddles, monitoring was simply discontinued. We observed in many cases that, while these patients may have had legitimate indications for monitoring upon admission, their conditions had improved; after brief multidisciplinary discussion, the team concluded that monitoring was no longer indicated. This observation may suggest interventions at the ordering phase, such as prespecifying a monitoring duration. ${ }^{22,23}$

This study's findings were consistent with a quasi-experimental study of safety huddle-based alarm discussions in a pediatric intensive care unit that showed a patient-level reduction of 116 alarms per patient-day in those discussed in huddles relative to controls. ${ }^{11}$ A smaller quasi-experimental study of implementing a nighttime alarm "ward round" in an adult intensive care unit showed a significant reduction in unit-level alarms/patient-day from 168 to $84 .^{9}$ In a quality improvement report, a monitoring care process bundle that included discussion of alarm settings showed a reduction in unit-level alarms/ patient-day from 180 to $40 .{ }^{10}$ Our study strengthens the body of literature using a cluster-randomized design, measuring patient- and unit-level outcomes, and including implementation outcomes that explain effectiveness findings.

On a hypothetical unit similar to the ones we studied with 20 occupied beds and 60 alarms/patient-day, an average of 1,200 alarms would occur each day. We delivered the intervention to 0.85 patients per day. Changes were made at the bedside in $60 \%$ of those with the intervention delivered, and those patients had a difference in differences of 119 fewer alarms compared with the comparison patients on control units. In this scenario, we could expect a relative reduction of $0.85 \times 0.60$ $x 119=61$ fewer alarms/day total on the unit or a $5 \%$ reduction. However, that estimated reduction did not account for the arrival of new patients with high alarm rates, which certainly occurred in this study and explained the lack of effect at the unit level.

As described above, the intervention dose was low, which translated into a lack of effect at the unit level despite a strong effect at the patient level. This result was partly due to the manual process required to produce the alarm dashboards that restricted their availability to nonholiday weekdays. The study was performed at one hospital, which limited generalizability. The study hospital was already convening daily safety huddles that were well attended by nurses and physicians. Other hos- 
pitals without existing huddle structures may face challenges in implementing similar multidisciplinary alarm discussions. In addition, the study design was randomized at the unit (rather than patient) level, which limited our ability to balance potential confounders at the patient level.

\section{CONCLUSION}

A safety huddle intervention strategy to drive alarm customization was effective in safely reducing alarms for individual children discussed. However, unit-level alarm rates were not affected by the intervention due to a low dose. Leaders of efforts to reduce alarms should consider beginning with passive interventions (such as changes to default settings and alarm delays) and use huddle-based discussion as a second-line intervention to address remaining patients with high alarm rates.

\section{Acknowledgments}

We thank Matthew MacMurchy, BA, for his assistance with data collection.

Funding/Support: This study was supported by a Young Investigator Award (Bonafide, PI) from the Academic Pediatric Association.

Trial Registration: ClinicalTrials.gov identifier: NCT02458872. https://clinicaltrials.gov/ct2/show/NCT02458872

Role of the Funder/Sponsor: The Academic Pediatric Association had no role in the design or conduct of the study; collection, management, analysis, or interpretation of the data; preparation, review, or approval of the manuscript; or decision to submit for publication.

Disclosures: No relevant financial activities, aside from the grant funding from the Academic Pediatric Association listed above, are reported.

\section{References}

1. Schondelmeyer AC, Brady PW, Goel W, et al. Physiologic monitor alarm rates at 5 children's hospitals. J Hosp Med. 2018;13(8):396-398.

2. Cvach $M$, Kitchens $M$, Smith $K$, Harris P, Flack MN. Customizing alarm limits based on specific needs of patients. Biomed Instrum Technol. 2017;51(3): 227-234.

3. Bonafide CP, Lin R, Zander M, et al. Association between exposure to nonactionable physiologic monitor alarms and response time in a children's hospital. J Hosp Med. 2015;10(6):345-351.

4. Bonafide CP, Localio AR, Holmes JH, et al. Video analysis of factors associated with response time to physiologic monitor alarms in a children's hospital. JAMA Pediatr. 2017;171(6):524-531.

5. Lange K, Nowak M, Zoller R, Lauer W. Boundary conditions for safe detection of clinical alarms: An observational study to identify the cognitive and perceptual demands on an Intensive Care Unit. In: In: D. de Waard, K.A. Brookhuis, A. Toffetti, A. Stuiver, C. Weikert, D. Coelho, D. Manzey, A.B. Ünal, S. Röttger, and N. Merat (Eds.) Proceedings of the Human Factors and Ergonomics Society
Europe Chapter 2015 Annual Conference. Groningen, Netherlands; 2016.

6. Westbrook JI, Li L, Hooper TD, Raban MZ, Middleton S, Lehnbom EC. Effectiveness of a 'Do not interrupt' bundled intervention to reduce interruptions during medication administration: a cluster randomised controlled feasibility study. BMJ Qual Saf. 2017;26:734-742.

7. Chopra V, McMahon LF Jr. Redesigning hospital alarms for patient safety: alarmed and potentially dangerous. JAMA. 2014;311(12):1199-1200.

8. Turmell JW, Coke L, Catinella R, Hosford T, Majeski A. Alarm fatigue: use of an evidence-based alarm management strategy. J Nurs Care Qual. 2017:32(1):47-54

9. Koerber JP, Walker J, Worsley M, Thorpe CM. An alarm ward round reduces the frequency of false alarms on the ICU at night. $J$ Intensive Care Soc. 2011;12(1):75-76.

10. Dandoy $C E$, Davies $S M$, Flesch $L$, et al. A team-based approach to reducing cardiac monitor alarms. Pediatrics. 2014;134(6):e1686-1694.

11. Dewan $M$, Wolfe $H$, Lin $R$, et al. Impact of a safety huddle-based intervention on monitor alarm rates in low-acuity pediatric intensive care unit patients. J Hosp Med. 2017;12(8):652-657.

12. Goldenhar LM, Brady PW, Sutcliffe KM, Muething SE. Huddling for high reliability and situation awareness. BMJ Qual Saf. 2013;22(11):899-906.

13. Brady PW, Muething $S$, Kotagal $U$, et al. Improving situation awareness to reduce unrecognized clinical deterioration and serious safety events. Pediatrics. 2013;131:e298-308.

14. Curran GM, Bauer M, Mittman B, Pyne JM, Stetler C. Effectiveness-implementation hybrid designs: combining elements of clinical effectiveness and implementation research to enhance public health impact. Med Care. 2012;50(3):217-226.

15. Damschroder LJ, Aron DC, Keith RE, Kirsh SR, Alexander JA, Lowery JC. Fostering implementation of health services research findings into practice: a consolidated framework for advancing implementation science. Implement Sci. 2009;4(1):50

16. Proctor E, Silmere H, Raghavan R, et al. Outcomes for implementation research: conceptual distinctions, measurement challenges, and research agenda. Adm Policy Ment Health. 2011;38(2):65-76.

17. Allen JD, Linnan LA, Emmons KM. Fidelity and its relationship to implementation effectiveness, adaptation, and dissemination. In: Dissemination and Implementation Research in Health: Translating Science to Practice (Brownson RC, Proctor EK, Colditz GA Eds.). Oxford University Press; 2012:281-304.

18. Harris PA, Taylor R, Thielke R, Payne J, Gonzalez N, Conde JG. Research electronic data capture (REDCap)—a metadata-driven methodology and workflow process for providing translational research informatics support. J Biomed Inf. 2009;42:377-381

19. Singer JD, Willett JB. Applied Longitudinal Data Analysis: Modeling Change and Event Occurrence. New York: Oxford University Press; 2003.

20. Wagner AK, Soumerai SB, Zhang F, Ross-Degnan D. Segmented regression analysis of interrupted time series studies in medication use research. $J$ Clin Pharm Ther. 2002;27:299-309.

21. Gardner W, Mulvey EP, Shaw EC. Regression analyses of counts and rates: Poisson, overdispersed Poisson, and negative binomial models. Psychol Bull. 1995; 118:392-404.

22. Dressler R, Dryer MM, Coletti C, Mahoney D, Doorey AJ. Altering overuse of cardiac telemetry in non-intensive care unit settings by hardwiring the use of American Heart Association guidelines. JAMA Intern Med. 2014;174(11):1852-1854.

23. Boggan JC, Navar-Boggan AM, Patel V, Schulteis RD, Simel DL. Reductions in telemetry order duration do not reduce telemetry utilization. J Hosp Med. 2014;9(12):795-796. 